\title{
Effects of Aerobic Exercise on Fasting Blood Glucose and Blood Pressure Levels of Diabetic-Hypertensive Clients at a Diabetes Clinic in Accra, Ghana
}

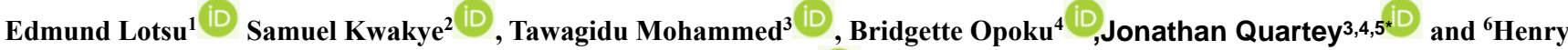 \\ Lawson $^{6}$ iD
}

\begin{abstract}
${ }^{1}$ Physiotherapy Department, Korle-Bu Teaching Hospital, Accra, Ghana. ${ }^{2}$ West Africa Football Academy, Sogakope, Ghana. ${ }^{3}$ Department of Physiotherapy, School of Biomedical and Allied Health Sciences, College of Health Sciences, University of Ghana, Accra, Ghana. ${ }^{4}$ School of Health Professions Education, Faculty of Health, Medicine and Life Sciences, Maastricht University, Netherlands. ${ }^{5}$ Centre for Sport, Exercise and Osteoarthritis Research Versus Arthritis, UK. ${ }^{6}$ Department of Community Health, University of Ghana Medical School, College of Health Sciences, University of Ghana.
\end{abstract}

*Corresponding author: neeayree@googlemail.com

\section{Abstract}

To cite: Lotsu E, Kwakye S, Mohammed T, Opoku B, Quartey J, Lawson H, Effects of Aerobic Exercise on Fasting Blood Glucose and Blood Pressure Levels of Diabetic-Hypertensive Clients at a Diabetes Clinic in Accra, Ghana. JPRM 2021, 3(2): 75-84. doi: 10.21617/jprm2021.3213

Background: Hypertension and diabetes are associated with an increased risk of cardiovascular diseases. Effective interventions are therefore relevant in reducing the morbidity and mortality associated with hypertension and diabetes. We set out to determine the effect of aerobic exercise on type-2 diabetic-hypertensive clients at a diabetes clinic in Accra.

Method: Twenty-one participants were recruited from the National Diabetes Management and Research Center in Korle-Bu in Accra. Participants undertook 30 minutes of aerobic exercise comprising, warm up, ergonomic cycling and cool down, three times a week for a period of eight weeks. Fasting blood glucose and blood pressure levels were measured and changes with respect to exercise were analysed. The mixed model ANOVA was used to test for the differences in the weekly blood pressure and blood glucose levels while the paired t-test was used to compare the baseline and final readings of blood pressure and blood glucose levels. Pearson's correlation test was used to determine the relationship between age and change in blood pressure and blood glucose levels after eight weeks of exercise.

Results: Mean systolic blood pressure reduced from $142 \mathrm{mmHg}$ to $135 \mathrm{mmHg}$ while the mean diastolic blood pressure also reduced from $89 \mathrm{mmHg}$ to $84 \mathrm{mmHg}$ post exercises. The baseline and eighth week post exercise average blood glucose level were $8.0 \mathrm{mmol} / \mathrm{L}$ and $5.2 \mathrm{mmol} / \mathrm{L}$ respectively. There were significant differences in baseline and week eight systolic $(\mathrm{p}=0.0017)$, and diastolic $(\mathrm{p}=0.006)$ blood pressure as well as blood glucose $(\mathrm{p}=0.0027)$ levels. There was also a significant positive correlation between age and change in blood glucose level $(p=0.036)$.

Conclusion: Aerobic exercise reduces the fasting blood glucose level and blood pressure in type-2 diabetichypertensives. Diabetic-hypertensives should therefore be encouraged to participate in aerobic exercises.

Keywords: aerobic exercise, blood glucose level, diabetic-hypertensive, blood pressure, clients. 


\section{INTRODUCTION}

Hypertension is a common cardiovascular disease responsible for 9.4 million deaths each year, or $16.5 \%$ of all deaths worldwide and it is defined as persistent elevation of systolic blood pressure of $140 \mathrm{mmHg}$ or greater and/or diastolic blood pressure of $90 \mathrm{mmHg}$ or greater [1]. As of 2000 , nearly one billion people or approximately $26 \%$ of the adult population of the world were living with hypertension [2]. Aerobic exercise is almost completely free of secondary effects and is a useful adjunct therapy for treating hypertension [3]. An immediate (acute) reduction in blood pressure following exercise has been termed 'post-exercise hypotension' and is caused by reductions in vascular resistance [4]. There is evidence that aerobic exercises help to reduce blood pressure. Molmen-Hansen et al. [5] in their study of 39 women with essential hypertension randomized for aerobic interval training indicated that the blood pressure reducing effect of exercise is intensity dependent and hence, the exercise mode used is an effective method to lower blood pressure and improve other cardiovascular risk factors.

Diabetes mellitus is characterized by abnormalities in insulin production and action or both, and its prevalence in UK varies from $2.7 \%$ to $4.1 \%$ of the population [6]. Living with diabetes means coping with a regimen of dietary management, physical exercise and periodic testing [6]. Exercise as a key prevention strategy for diabetes is commonly accepted and recommended throughout the world [7]. Unfortunately, not all individuals profit to the same extent as some exhibit exercise resistance [7]. Aerobic exercise, with cardiovascular equipment like treadmill, improves the blood glucose level and decreases the free radicals in patients with diabetes mellitus [8]. Reduction in blood glucose levels during exercise may be due to the conversion of blood glucose to glycogen to produce energy during aerobic exercise [9]. Moderate-intensity exercise improves blood glucose (BG), but most people fail to achieve the required exercise volume [10]. Although, highintensity exercise (HIE) protocols may vary, very brief HIE improves blood glucose one to three days post exercise in both diabetics and nondiabetics [10].

The generally high prevalence of hypertension is currently driven by two phenomena: the increased age of the population and the growing prevalence of obesity, which is seen in developing as well as developed countries [11]. With current research that relates the occurrence of hypertension with diabetes and the beneficial effects of exercises in both diseases [12, 13], it has become imperative to know or ascertain how aerobic exercises specifically affect diabetichypertensive patients. Diabetic-hypertensive patient is an individual diagnosed with both type 2 diabetes and hypertension.

Empirical evidence shows that physiotherapy has not been fully involved in the management of diabetic-hypertensive patients in Ghana. The practice of referral of such patients for physiotherapy is minimal in spite of documented potential benefits of aerobic exercise on both diabetes and hypertension and promotion of overall health indicate this. Moreover, there is a paucity of literature in Ghana on the effects of aerobic exercise on fasting blood glucose and blood pressure levels in diabetic- hypertensives. Thus, in view of the renewed concern to improve health conditions of people living with both diabetes and hypertension through the most costeffective means, this study was designed to determine the effects of aerobic exercise on such people (type-2 diabetic-hypertensive clients). METHODS AND MATERIALS

This quasi experimental pilot research was conducted at the National Diabetes Management and Research Center (NDMRC), Korle-Bu Teaching Hospital, Accra, Ghana. All ambulant individuals between 45 and 65 years, diagnosed with type-2 diabetes and hypertension referred to NDMRC were recruited via the simple random sampling method with participants for each day selected by picking one of two letters (A or B) to be included in the study or otherwise. At least two participants were recruited on each of the three clinic days in a week over a three-week period. A total of 25 individuals who agreed to participate in the study were recruited. The sample size was determined by the formula:

$\mathrm{n}=2(\mathrm{Z1}+\mathrm{Z2}) 2 . \delta 2 / \mathrm{d} 2$ [14], where $\mathrm{n}$ $=$ sample size, $Z 1=\mathrm{z}$-value at $\alpha=0.005=1.96$, $\mathrm{Z} 2=\beta=0.20=0.84, \delta=$ standard deviation, estimated to be 5 and $d=$ change in score was estimated at 4.8 due to the clinical significance of such studies as showed by Maggard et al [14]. Thus, $\mathrm{n}=2(1.96+0.84) 2 * 52 / 4.82=17.01$. A minimum sample size of 17 was therefore determined. Patients with gestational diabetes and or communication and cognitive impairments such as Aphasia were excluded from this study. Patients with co-morbidities such as cancer that may be contraindicated to exercise were also excluded from the study.

The instruments used for data collection were a mercury sphygmomanometer 
(PARAMED brand), stethoscope (Littmann Classic brand), ergometric cycle (ERG 910 Plus brand) and blood glucose meter (Freestyle Lite brand). The sphygmomanometer and a stethoscope were used to measure participants' blood pressure in millimetres of mercury $(\mathrm{mmHg})$ and the ergometric cycle used for aerobic exercises on eligible clients. The blood glucose meter was used to measure the amount of glucose in blood millimoles per litre $(\mathrm{mmol} / \mathrm{L})$.

Demographic data and other information (age, gender, medications, time of last meal [onset of the study] and acute illnesses) relating to their condition was obtained from their medical folders through the Head of the NDMRC. A biomedical laboratory scientist assisted in training the researchers to measure fasting blood glucose level using a glucometer. The blood pressure was measured with a stethoscope and sphygmomanometer. Data collection commenced in August 2013 and ended in February 2014.

Baseline tests were measured on the first day of assessment by the researchers before engaging the patients in the exercise protocol. On each day of exercise, the blood pressure and blood glucose levels were established before exercises began. Patients' medications were continued throughout.

Participants were taken through 30 minutes of exercise (aerobics and ergometric cycling) for eight (8) weeks uninterrupted with an intensity of approximately $25 \%$ of $\mathrm{VO} 2$ max (the highest oxygen delivered and utilized during exercise) [15], which included warmups and cool downs, relative to the patients' abilities three times a week. Participants were required in consultation with the physicians in charge to continue their medications throughout the data collection period. The exercises were carried out in the gymnasium of the Physiotherapy Department of Korle-Bu Teaching Hospital. Patients fasting blood glucose and blood pressure levels were measured after exercising for every visit until the 8 th week.

Data was analyzed using SPSS. Descriptive statistics of means and standard deviations were used to represent changes in blood glucose and blood pressure levels. The mixed model ANOVA was used to test for the differences in the weekly blood pressure and blood glucose levels. The paired t-test was used to compare the baseline and final readings of blood pressure and glucose levels. Pearson's correlation test was used to determine the relationship between age and change in blood pressure and glucose levels after eight weeks of exercise. The level of significance was set at $p$ $=0.05$.

\section{RESULTS}

Twenty-five participants were recruited for the study of which 21 participated actively giving a response rate of $84 \%$. Some of the 21 participants skipped some sessions due to high glucose or BP levels or inability to turn up due to genuine reasons. However, these participants completed the eight-week exercise programme which gave rise to some missing values. Expectation maximization (EM) analysis was used to construct and analyze the missing values. The parameter of interest was estimated in each dataset separately, and combined. Imputed values compared reasonably to observed values and results using the complete case analysis were similar to EM. Eight (38.1\%) participants were females and 13 (61.9\%) were males. Majority 15 (71.4\%) of participants were between the ages of 45 and 58 years with a mean age of $55(\square 7)$ years.

There was reduction of $7 \mathrm{mmHg}$ (from $142 \mathrm{mmHg}$ to $135 \mathrm{mmHg}$ ) in mean systolic pressure with a spurt $(128 \mathrm{mmHg}$ and $132 \mathrm{mmHg})$ in average systolic blood pressure between the sixth and seventh weeks followed by a reduction to $129 \mathrm{mmHg}$. There was also a reduction of $5 \mathrm{mmHg}$ (from $89 \mathrm{mmHg}$ to $84 \mathrm{mmHg}$ ) in mean diastolic blood pressure. There was however a decreasing and increasing trend in average diastolic blood pressure from baseline to last measurements with a spurt $(79 \mathrm{mmHg}$ to $85 \mathrm{mmHg}$ ) between the seventh and eighth measurement before it dropped to $84 \mathrm{mmHg}$ at the final measurement.

The baseline and eight weeks post exercise average blood glucose level of participants was $8.0 \mathrm{mmol} / \mathrm{L}$ and $5.2 \mathrm{mmol} / \mathrm{L}$ respectively. A mean reduction of $1.4 \mathrm{mmol} / \mathrm{L}$ ( $\square$ 1.4) over the eight-week period was observed as shown in Table 1. 
Lotsu E, Kwakye S, Mohammed T, Opoku B, Quartey J, Lawson H, Effects of Aerobic Exercise on Fasting Blood Glucose and Blood Pressure Levels of Diabetic-Hypertensive Clients at a Diabetes Clinic in Accra, Ghana.

Table 1: Distribution of mean blood glucose levels from baseline to week eight

\begin{tabular}{|c|c|c|c|c|c|c|c|c|c|c|}
\hline & \multicolumn{5}{|c|}{ Complete case analysis } & & \multicolumn{4}{|c|}{ Expectation maximization analysis } \\
\hline Week & $\mathrm{N}$ & $\min$ & $\max$ & Mean & Std & $\mathrm{N}$ & Min & $\max$ & Mean & std \\
\hline Baseline & 21 & 4.3 & 13.6 & 8.0 & 2.8 & 21 & 4.3 & 13.6 & 8.0 & 2.8 \\
\hline 1 & 21 & 4.5 & 13.0 & 7.4 & 2.1 & 21 & 4.5 & 13.0 & 7.4 & 2.1 \\
\hline 2 & 21 & 4.9 & 11.0 & 7.1 & 1.7 & 21 & 4.9 & 11.0 & 7.1 & 1.7 \\
\hline 3 & 14 & 4.9 & 8.0 & 6.1 & 1.0 & 21 & 4.9 & 8.0 & 6.1 & 0.8 \\
\hline 4 & 13 & 4.5 & 9.3 & 6.5 & 1.6 & 21 & 4.5 & 9.3 & 6.5 & 1.2 \\
\hline 5 & 18 & 4.2 & 9.0 & 5.9 & 1.2 & 21 & 4.2 & 9.0 & 5.9 & 1.1 \\
\hline 6 & 18 & 4.2 & 8.4 & 5.6 & 1.1 & 21 & 4.2 & 8.4 & 5.6 & 1.0 \\
\hline 7 & 15 & 4.2 & 8.2 & 5.5 & 1.0 & 21 & 4.2 & 8.2 & 5.5 & 0.8 \\
\hline 8 & 5 & 4.0 & 7.5 & 5.2 & 1.4 & 21 & 4.0 & 7.5 & 5.2 & 0.6 \\
\hline
\end{tabular}

$\mathrm{N}=$ Number of participants

There was a significant change of the weekly effect on systolic $(\mathrm{p}=0.011)$ and diastolic $(\mathrm{p}=0.042)$ blood pressure of the weekly differences in blood pressure levels on performing the aerobic exercises and ergometric cycling as shown in Table 2.

Table 2: Weekly effect of exercises on blood pressure levels

\begin{tabular}{|l|l|l|l|l|}
\hline \multicolumn{2}{|l|}{} & \multicolumn{2}{l|}{ Diastolic blood pressure } \\
\hline Source & F & P-value & F & P-value \\
\hline Intercept & 12971.421 & 0.000 & 12551.797 & 0.000 \\
\hline Week & 2.597 & $0.011^{\star}$ & 1.917 & $0.042^{*}$ \\
\hline
\end{tabular}

* =statistically significant 
The systolic blood pressure has an upper and lower bound of $148 \mathrm{mmHg}$ and $137 \mathrm{mmHg}$ respectively for baseline measurement while the week eight upper and lower bound measurements are $140 \mathrm{mmHg}$ and $118 \mathrm{mmHg}$ respectively as shown in Table 3 .

Table 3: Systolic blood pressure distribution from baseline to week-eight

\begin{tabular}{|c|c|c|c|c|c|c|}
\hline & \multicolumn{3}{|c|}{ Complete case analysis } & \multicolumn{3}{|c|}{ Expectation maximization analysis } \\
\hline & \multicolumn{3}{|c|}{ Systolic blood pressure } & \multicolumn{3}{|c|}{ Systolic blood pressure } \\
\hline Week & $\mathrm{N}$ & Mean \pm SD & $95 \% \mathrm{Cl}$ & $\mathrm{N}$ & Mean \pm SD & $95 \% \mathrm{Cl}$ \\
\hline Baseline & 21 & $142 \pm 20$ & $137-148$ & 21 & $142 \pm 20$ & $137-148$ \\
\hline 1 & 21 & $140 \pm 14$ & $134-145$ & 21 & $140 \pm 14$ & $134-145$ \\
\hline 2 & 21 & $138 \pm 9$ & $132-143$ & 21 & $138 \pm 9$ & $132-143$ \\
\hline 3 & 21 & $137 \pm 12$ & $130-144$ & 21 & $137 \pm 12$ & $130-144$ \\
\hline 4 & 14 & $132 \pm 14$ & $125-139$ & 21 & $132 \pm 11$ & $125-139$ \\
\hline 5 & 13 & $130 \pm 10$ & $124-136$ & 21 & $130 \pm 9$ & $124-136$ \\
\hline 6 & 18 & $128 \pm 11$ & $122-134$ & 21 & $128 \pm 10$ & $122-134$ \\
\hline 7 & 18 & $132 \pm 9$ & $125-138$ & 21 & $132 \pm 8$ & $125-138$ \\
\hline 8 & 15 & $129 \pm 5$ & $118-140$ & 21 & $129 \pm 5$ & $118-140$ \\
\hline
\end{tabular}

The diastolic blood pressure shows an upper and lower bound of $93 \mathrm{mmHg}$ and $85 \mathrm{mmHg}$ respectively for baseline measurement while week eight shows an upper and lower bound measurement are $91 \mathrm{mmHg}$ and $77 \mathrm{mmHg}$ respectively which are all shown in Table 4 . 
Lotsu E, Kwakye S, Mohammed T, Opoku B, Quartey J, Lawson H, Effects of Aerobic Exercise on Fasting Blood Glucose and Blood Pressure Levels of Diabetic-Hypertensive Clients at a Diabetes Clinic in Accra, Ghana.

Table 4: Diastolic blood pressure distribution from baseline to week 8

\begin{tabular}{|l|l|l|l|l|l|l|l|}
\hline \multicolumn{4}{|l|}{ Complete case analysis } & & \multicolumn{3}{l|}{ Expectation maximization analysis } \\
\hline & \multicolumn{2}{|l|}{ Diastolic blood pressure } & & \multicolumn{3}{l|}{ Diastolic blood pressure } \\
\hline Week & $\mathrm{N}$ & Mean \pm SD & $95 \% \mathrm{Cl}$ & & $\mathrm{N}$ & Mean \pm SD & $95 \% \mathrm{Cl}$ \\
\hline Baseline & 21 & $97 \pm 3$ & $82-111$ & & 21 & $97 \pm 3$ & $82-111$ \\
\hline 1 & 21 & $93 \pm 3$ & $79-107$ & & 21 & $93 \pm 3$ & $79-107$ \\
\hline 2 & 21 & $93 \pm 3$ & $79-107$ & & 21 & $93 \pm 3$ & $79-107$ \\
\hline 3 & 21 & $87 \pm 9$ & $48-124$ & & 21 & $81 \pm 2$ & $78-85$ \\
\hline 4 & 14 & $87 \pm 3$ & $72-101$ & & 21 & $85 \pm 1$ & $82-87$ \\
\hline 5 & 13 & $87 \pm 3$ & $72-101$ & & 21 & $84 \pm 1$ & $82-87$ \\
\hline 6 & 18 & $83 \pm 3$ & $67-98$ & & 21 & $79 \pm 1$ & $76-82$ \\
\hline 7 & 18 & $87 \pm 3$ & $72-101$ & & 21 & $85 \pm 2$ & $81-88$ \\
\hline 8 & 15 & $83 \pm 3$ & $67-98$ & & 21 & $84 \pm 1$ & $82-85$ \\
\hline
\end{tabular}

There were significant differences in baseline and final (week eight) systolic $(p=0.0017)$, diastolic $(p=0.006)$ blood pressure as well as blood glucose $(p=0.0027)$ levels as shown in Table 5.

Table 5: Comparison of baseline and week eight differences in blood pressure and glucose levels

\begin{tabular}{|c|c|c|c|c|c|}
\hline \multirow[b]{2}{*}{ Item } & \multirow[b]{2}{*}{ Mean } & \multicolumn{2}{|c|}{ Paired Differences } & \multirow[b]{2}{*}{$\mathbf{T}$} & \multirow[b]{2}{*}{$\begin{array}{c}\text { Sig } \\
\text { (2-tailed) }\end{array}$} \\
\hline & & $\begin{array}{c}\text { Std. } \\
\text { Deviation }\end{array}$ & $\begin{array}{c}\text { Std. Error } \\
\text { Mean }\end{array}$ & & \\
\hline Systolic blood pressure: & 12.047 & 21.214 & 4.629 & 2.602 & $0.017^{*}$ \\
\hline Diastolic blood pressure: & 7.142 & 10.555 & 2.303 & 3.101 & $0.006^{*}$ \\
\hline Blood glucose Level: & 2.366 & 2.972 & 0.648 & 3.649 & $0.002^{*}$ \\
\hline
\end{tabular}

* = statistically significant $\quad$ Std $=$ Standard $\quad$ Sig $=$ Significant

There was a significant correlation between age and change in blood glucose level $(p=0.036)$. However, there was no correlation between age and change in systolic blood pressure $(p=0.453)$ as well as age and change in diastolic blood pressure $(p=0.453)$ as shown in Table 6 . 
Table 6: Relationship between age and change in blood glucose and pressure levels

\begin{tabular}{|c|c|c|c|c|}
\hline & & $\begin{array}{c}\text { Week 1- } \mathbf{8} \\
\text { Overall mean change in } \\
\text { SBP }\end{array}$ & $\begin{array}{c}\text { Week 1-8 } \\
\text { Overall mean } \\
\text { change in DBP }\end{array}$ & $\begin{array}{c}\text { Week 1-8 } \\
\text { Overall mean change in } \\
\text { Glucose Level }\end{array}$ \\
\hline \multirow{3}{*}{ Age } & Pearson Correlation & -0.173 & -0.143 & -0.460 \\
\hline & P-value & 0.453 & 0.535 & 0.036 \\
\hline & $\mathrm{N}$ & 21 & 21 & 21 \\
\hline
\end{tabular}

\section{DISCUSSION}

This study revealed that there were more men than women. The findings of this study corroborate findings of Siddiqi et al. [6], who reported that diabetes was more prevalent in males than in females but contrary to findings of Kearney et al. [2] who indicated that the prevalence of diabetes and hypertension in women was more than men. This indicates a prevalence of the combination of diabetes and hypertension in men although this was is inconsequential for the design of this study. Although the difference in gender regarding the number of diabetic-hypertensives in this study was not huge, the reason for a contrary outcome with Keaney et al. [2], may be attributed to the sample size of this study and the fact that this study was conducted among diabetichypertensives whereas Keaney et al. conducted theirs with diabetic and hypertensive patients. Aerobic exercise is and can be performed by both male and female thus did not impose any form of restriction to participants. The study was rather meant to inform or direct the use of aerobic exercise as an intervention for diabetichypertensives irrespective of gender. Patients also reported a significant increase in general body strength and mood, which may be considered an added benefit of aerobic exercise. The increase in general body strength may also be due to increased blood circulation to the musculoskeletal system, which helps in muscle nutrition and contractility [16]. This may be attributed to the general effect of exercise especially cycling which includes increased general body strength, energy and increased level of endorphins, which are natural mood lifters [17]. Cardoso et al. [18] reported improved cardiorespiratory fitness, concomitant with increased participation in physical activity and decreased fatigue severity, following an aerobic exercise-training regimen which included treadmill walking for 30-45 minutes per session over 10 -weeks. Specific characteristics of the exercise itself might also be important in determining post-exercise hypotension and general body strength. However, the effect of exercise intensity still remains controversial [16]. For example, in this study participants in this study, participants went through. Although the exercise regimen (30 minutes of aerobics and ergometric cycling for eight weeks uninterrupted with an intensity of approximately $25 \%$ of $\mathrm{VO} 2$ max) used in this study may have different characteristics and intensity similar results were achieved as in other studies with relatively different exercise regimen as reported by Cardoso et al. [18].

The reduced blood pressure levels of all participants after eight weeks of aerobic exercise compared to values obtained at baseline corroborate the outcomes of Molmen et al. [5] who reported that aerobic interval training was an effective method that lowers blood pressure and improves other cardiovascular risk factors. The extent of exercise-induced reduction of blood pressure varies from 5 to $15 \mathrm{mmHg}$ among studies [19] while in older hypertensives such as participants of this study, the effect is not as pronounced in younger individuals and is reported to be 5 to $6 \mathrm{mmHg}$. Thus, the present study's systolic blood pressure reduction of $7 \mathrm{mmHg}$ in diabetic-hypertensives is not lower than the anticipated range for hypertension. Radhakrishnan and Ekambaram [19] revealed that acute aerobic exercise is able to reduce ambulatory blood pressure levels when these levels are already elevated, whereas chronic aerobic exercise can reduce ambulatory blood pressures in normotensive and especially hypertensive subjects. Thus, aerobic training is a very useful tool for the prevention and treatment of hypertension. This may be due to an immediate (acute) reduction in blood pressure termed 'post- 
exercise hypotension' which is caused by reductions in vascular resistance [17]. In order to be clinically relevant, post-exercise hypotension must have a significant magnitude and be sustained for a long period of time under ambulatory conditions [19].

The significant difference observed among the weekly average glucose levels and the reduction in the upper and lower bound levels after eight weeks may be due to the conversion of blood glucose to glycogen to produce energy during the prescribed aerobic exercise [9] which corroborates outcomes by Anja et al. [7] who reported that there was significant reduction in fasting blood glucose levels in patients with type2 diabetes who undertook aerobic exercise. Since muscle contraction increases glucose uptake in skeletal muscles, physical activity has been suggested in type-2 diabetes mellitus [20]. Moderate levels of aerobic exercises were performed by participants of this study since it affects a large group of muscles over time, which led to reduction in fasting blood sugar, after the eight weeks of exercise intervention. This effect is related to the promotion of glucose uptake in the skeletal muscles and loss of body fat in the body central part during exercise [24]. Yang et al. [16] reported decreases in glucose level and insulin resistance index after 12 weeks of aerobic exercise, resistance exercise and combined exercise in women with type 2 diabetes mellitus. However, these changes were bigger among the participants in the combined exercise group. Aminilari et al. [20] confirms that both resistance and aerobic exercises are effective in diabetes control by decreasing fasting blood sugar and glycosylated haemoglobin.

The comparison of baseline and final systolic blood pressure of this study was significantly different as also found by Ruivo and Alcântara [3]. A decrease of $7 \mathrm{mmHg}$ in systolic and $5 \mathrm{mmHg}$ in diastolic blood pressures as reported in this study, is of clinical relevance with regard to cardiovascular risk as demonstrated by Dimeo [21]. Mohan et al. [22] showed that aerobic exercise significantly decreased systolic and diastolic daytime ambulatory blood pressure by $6 \pm 12$ and $3 \pm 7 \mathrm{mmHg}$, respectively among resistant hypertensives and concluded that physical exercise is able to decrease blood pressure even in persons with low responsiveness to medical treatment. Post-exercise hypotension" may be the reason for reduction in systolic blood pressure. This is because during exercise, there is a decrease in the peripheral vascular resistance and thus an overall reduction in blood pressure [23].
There was no correlation between age and change in systolic blood pressure which suggest that older persons probably tend to have small or decreased changes in systolic blood pressure. Weinstein et al. [17] reported no correlation between age and hypertension but on the contrary, Weber et al. [11] revealed an increased systolic blood pressure after age 50 or 60 years. The difference in findings may be attributed to the category of participants used for the studies. This study was conducted among diabetic-hypertensives while Webber et al. [11] used apparently healthy individuals. Moreover, age probably represents an accumulation of environmental influences and the effect of genetically programmed senescence in body systems [21]. There was a negative correlation between age and change regarding diastolic blood pressure, thus older persons tend to have small changes (decrease) in diastolic blood pressure, which tends to corroborate reports by Weber et al [11]. Although, diastolic blood pressure values fluctuated a bit over the eight-week period, there was an overall $5 \mathrm{mmHg}$ reduction at the end. This could be considered remarkable because it buttresses the success of exercise in reducing blood pressure.

There was a negative but significant correlation between age and change in glucose levels, similar to findings by Adams [10] and Aminilari et al. [16] where high-intensity exercise caused a reduction in fasting blood sugar of diabetics. The outcomes of this study probably indicate that the effect of exercises depends on various factors such as the type, intensity and frequency. In this study, the maximum effect of aerobic exercise on blood glucose appeared after 8 weeks although there were minute reductions in blood glucose weekly. For instance, Oberbach et al. [24] reported improvement in glucose metabolism and insulin resistance after four-week training aerobic training protocol which included 60 minutes of swimming. Bello et al. [24] also indicated that the therapeutic benefits of aerobic exercise include regulation of body weight, reduction of insulin resistance, enhancement of insulin sensitivity and glycaemic control after they found no significant difference in mean age and duration of type 2 diabetes mellitus patients. LIMITATIONS

The referral of diabetic-hypertensive patients to physiotherapy for aerobic exercises appears minimal in Ghana in spite of its documented potential benefits and promotion of overall health. However, the outcomes of this study may be not be exactly attributable to the effect of the exercises due to the small sample size 
and lack of a control group for appropriate comparison of indices measured under similar conditions asides age-matching. Changes in dietary habits, the continued use of their medication and possible changes with medication were also not controlled in this study.

\section{CONCLUSION}

This study reiterates reported outcomes of studies in other countries. This study showed that half an hour of aerobic exercises three times a week for eight weeks probably caused a mean reduction of $1.4 \mathrm{mmol} / \mathrm{L}$ in fasting blood glucose levels as well as marginal reduction in systolic and diastolic blood pressure in ambulant type-2 diabetic-hypertensives clients. Type-2 diabetichypertensives clients should therefore be encouraged to participate in at least half an hour of aerobic exercise thrice a week in conjunction with consistent education on their condition.

\section{DECLARATION}

Contributors Conceptualization: The concept and study design were developed by EL and JQ. EL, SK, TM, BO, JQ and $\mathrm{HL}$, were involved in data collection. $\mathrm{EL}, \mathrm{SK}$, and $\mathrm{JQ}$ provided advice on statistical analysis. EL, JQ, and $\mathrm{HL}$ contributed on the interpretation of results. The manuscript was written by EL, JQ and SK while TM, BO and $\mathrm{HL}$ provided substantial amounts of references. ES, SK, $\mathrm{TM}, \mathrm{BO}, \mathrm{JQ}$ and $\mathrm{HL}$ contributed towards the draft and approved the final manuscript.

Competing interests There were no competing interests from all authors in this study.

\section{Ethics approval}

Ethical clearance (ET. /10306805/AA/21A/2012-2013) was sought and obtained from the Ethics and Protocol Review Committee of the School of Allied Health Sciences, University of Ghana. Appropriate permissions and informed consent of the participants were obtained before recruitment into the study.

Acknowledgements Special thanks go to the participants recruited from the National Diabetes Management and Research Center of Korle Bu Teaching Hospital for their participation in this study. Appreciation also goes to the staff of the National Diabetes Management and Research Center, Physiotherapy Department of Korle Bu Teaching Hospital and the Department of Physiotherapy, School of Biomedical and Allied Health Sciences, University of Ghana for their support.

\section{REFERENCES}

1. "WHO Disease and injury country estimates". World Health Organization. 2011. Retrieved June. 11, 2019.
2. Kearney PM, Whelton M, Reynolds K, Whelton PK, He J. "Worldwide prevalence of hypertension: a systematic review". J. Hypertens 2004; 22 (1): 11-9.

3. Ruivo AJ \& Alcantara P. Hypertension and exercise. Revista Portuguesa de Cardiologia 2012; 31(2): 151-158.

4. de Brito LC, Rezende RA, da Silva Junior ND et al. Post-Exercise Hypotension and Its Mechanisms Differ after Morning and Evening Exercise: A Randomized Crossover Study. PLOS ONE 2015; 10(7): e0132458.

5. Molmen-Hansen HE, Stolen T., Tjonna AE, Aamot IL, Ekeberg IS, Tyldum GA., Stoylen A. Aerobic interval training reduces blood pressure and improves myocardial function in hypertensive patients. European Journal of Preventive Cardiology 2012, 19(2), 151-160.

6. Siddiqui MA, Khan MF, and Carline TE. Gender Differences in Living with Diabetes Mellitus. Mater Sociomed. 2013; 25(2): 140-142.

7. Anja B, Cora W, Harald S and Hans-Ulrich $\mathrm{H}$. Exercise and diabetes: relevance and causes for response variability, Endocrine 2016, 51(3), 390.

8. Jonker TJ, Mol P, Vries ST, Widya RL, Hammer S, Schinkel LD, et al. Exercise and Type 2 Diabetes Mellitus: Changes in Tissue-specific Fat Distribution and Cardiac Function. Radiology 2013; 269(2): 434-442.

9. Mergenthaler $\mathrm{P}$, Lindauer $\mathrm{U}$, Diene $\mathrm{GAl}$, and Meisel A. Sugar for the brain: the role of glucose in physiological and pathological brain function. Trends Neuroscience 2013; 36(10): 587-597.

10. Adams OP. The impact of brief high-intensity exercise on blood glucose levels. Diabetes Metab Syndr Obes. 2013; 6: 113-122.

11. Weber MA, Schiffrin EL, White WB et al. Clinical Practice Guidelines for the Management of Hypertension in the Community. Journal of clinical hypertension 2013; 16(1): 14-26.

12. Inder JD, Carlson DJ, Dieberg G, McFarlane JR, Hess $\mathrm{N}$ and Smart NA. Isometric exercise training for blood pressure management: a systematic review and meta-analysis to optimize benefit. Hypertension Research 2016; 39: 88-94.

13. Sheri R. Colberg SR, Sigal RJ, Yardley JA et al. Physical Activity/Exercise and Diabetes: A Position Statement of the American Diabetes Association. Diabetes Care 2016; 39(11): 20652079.

14. Maggard Ma, O'cornel, Lin Jh And Etzioni, (2003). Sample Size Calculations in Surgery: Are they Done Correctly? Surgery Vol 134, Issue2 Pages 275-279

15. Lubans DR, Morgan PJ, Callister R, Collins CE. The Relationship between Pedometer Step Counts and Estimated VO2Max as Determined by a Submaximal Fitness Test in Adolescents. 
Human Kinetics 2008, 20 (3): 273-284.

16. Yang Z, Scott C., Mao C., Tang J \& Farmer A. Resistance Exercise Versus Aerobic Exercise for Type 2 Diabetes: A Systematic Review and Meta-Analysis. Sports med 2014; 44: 487-499.

17. Weinstein A, Chin L., Keyser R., Kennedy M., Nathan S., Woolatenhulme $\mathrm{J}$ et al. Effect of aerobic exercise training on fatigue and physical activity in patients with pulmonary arterial hypertension. Respiratory Medicine 2013; 107: 778-784.

18. Cardoso CG, Gomides RS, Queiroz ACC, Pinto LG, Lobo FS, T Tinucci, et al. Acute and chronic effects of aerobic and resistance exercise on ambulatory blood pressure. Clinics 2010; 65(3): 317-325.

19. Radhakrishnan $S$ and Ekambaram M. Diabetes and hypertension prevalence among tribals. Archives of Medicine and Health Sciences 2015; 3(1), 66-71.

20. Aminilari Z., Fararouei M., Amanat S., Sinaei E et al. The Effect of 12 Weeks Aerobic, Resistance, and Combined Exercises on Omentin-1 Levels and Insulin Resistance among Type 2 Diabetic Middle-Aged Women. Diabetes Metab J 2017;41: 205-212.

21. Dimeo F, Pagonas N, Seibert F, Arndt R, Zidek W, Westhoff TH. Aerobic Exercise Reduces Blood Pressure in Resistant Hypertension. Hypertension 2012; 60: 653-658.

22. Mohan V, Deepa M, Farooq S, Datta M, Deepa R. Prevalence, awareness and control of hypertension in Chennai - The Chennai Urban Rural Epidemiology Study (CURES-52). J Assoc Physicians India 2007;55: 326-32.

23. Mancia G, Fagard R, Narkiewicz K, Redon J, Zanchetti A, Böhm M et al. (2013) ESH/ESC Guidelines for the management of arterial hypertension: The Task Force for the management of arterial hypertension of the European Society of Hypertension (ESH) and of the European Society of Cardiology (ESC), European Heart Journal 2013; 34(28): 21592219. https://doi.org/10.1093/eurheartj/eht151

24. Oberbach, A., Tonjes, A., Kloting, N., Fasshauer, M., Kratzsch, J., Busse, M.W et al. Effect of a 4week physical training program on plasma concentrations of inflammatory markers in patients with abnormal glucose tolerance. European Journal of Endocrinology 2006; 154(4): 577-585.

25. Bello A, Owusu-Boakye E, Adegoke B, Adjei D. Effects of aerobic exercise on selected physiological parameters and quality of life in patients with type 2 diabetes mellitus. International Journal of General Medicine 2011; 4: 723-727. 